\title{
A Speed and Distance Measuring Exercise for the Electrical Engineering Technology Laboratory
}

\author{
Russell A. Aubrey \\ Purdue University School of Technology, Anderson, Indiana
}

\begin{abstract}
Hands on exercises in introductory EET courses provide students with interesting instructional tools to pique their inquisitiveness and increase their knowledge. The application specific exercise being described was developed to provide students an experience connecting basic analog and digital circuits to produce a system for a specific application. While negotiating the path to the desired goal, students experience working with units of measure such as time, distance, velocity and associated math concepts. The exercise provides an opportunity for them to use light emitting diodes as a sender-receiver system in conjunction with basic circuits to measure the speed of an object passing between the sender-receivers. Additional information is obtained from the sensor system also allows the calculation of the object's width.
\end{abstract}

This paper describes the physical structure of the exercise, the circuits developed, a description of their layout and implementation and the methodology involved in their design. Laboratory problem solving application and calculations based on this exercise are described, and laboratory scenarios are presented.

Introduction

The purpose of a laboratory exercise in Electrical Engineering Technology (EET) is to provide students with hands on experience connecting components into circuits and taking data from those circuits. This exercise provides students the opportunity to connect basic analog and digital circuits to produce a usable electronic system that will measure the speed and width of a moving object.

Methodology

The target group for this exercise is first or second semester EET students. Therefore, the first criterion endorsed was that the exercise have an interest factor that would attract and hold the students' attention while providing a worthwhile laboratory experience. After a number of discussions with students and colleagues, the premise was established that the primary effort would be to measure the speed of an object passed between two vertical uprights. The measurement of "speed" had an interest factor for the students and the thought of measuring the speed of a "Karate Chop" soon evolved. A secondary thought developed that if speed and distance were part of the data set perhaps width measurement might be possible. 
Having settled on the basic concepts to be covered, the implementation technique began. To provide an exercise for first or second semester students, the circuits used were purposely restricted to the analog and digital circuits covered in these courses. Test equipment selected was of the type typically encountered in these courses. Options were included that allowed the exercise to run at various competence levels depending on the target group of students.

Circuit Implementation

The test stand where the sensing devices to measure the speed of the objects are mounted is shown in Figure 1. Infrared LED sender/receiver pairs are mounted six inches apart on the uprights as indicated. A multi wire cable connects the stand to the circuit board to provide power for the stand and return signals to the circuit board.

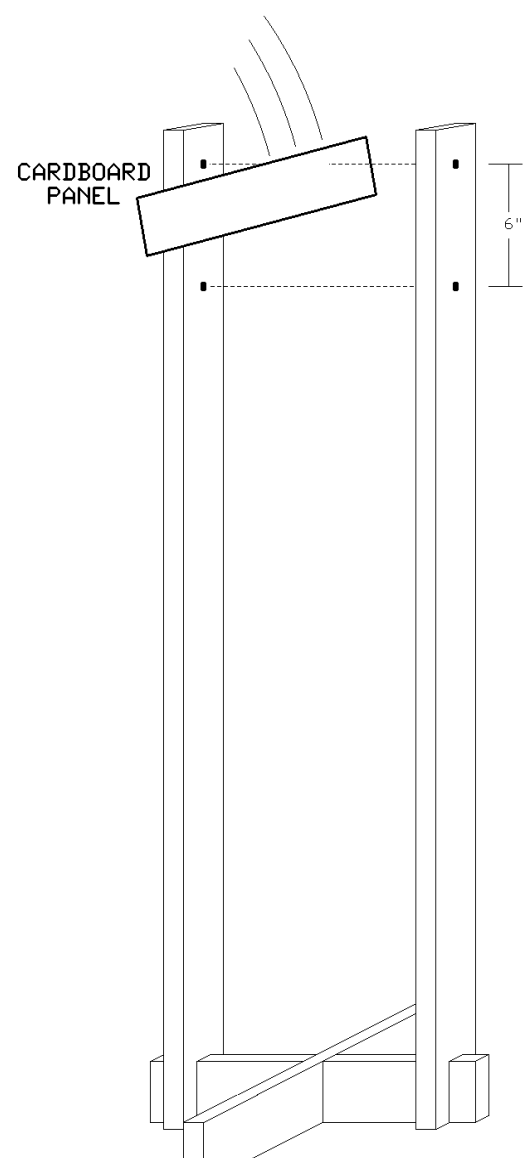

Figure 1 Test Stand

Shown in Figure 2 is the circuit used to measure the speed and width of an object passed through the beams as depicted in Figure 1. A $1,000 \mathrm{~Hz}$ time base is generated by the 555 -timer circuit and this pulse train is then gated into counters running in the totaling mode. The numbers appearing in the counters read directly in milliseconds which are utilized in the conversion techniques explained in the exercise handout.

As the top beam is broken by the object's leading edge, the U4a comparator circuit sends a pulse that sets the NAND latch. This NAND latch enables the pass gate to counter number two which then receives pulses from the time base generator. As the object's leading edge breaks the bottom beam, the U4b comparator circuit then resets the latch prohibiting additional pulses from reaching the counter. The reading on counter two is the total time in milliseconds elapsed while the object travels the fixed distance of six inches. This data allows the speed of the object to be calculated in inches per second (or any other desired ratio) as it passes through the uprights.

The same comparator outputs utilized above are also connected through inverters to the pass gates $\mathrm{U} 2 \mathrm{~b}$ and $\mathrm{U} 2 \mathrm{c}$. This allows the time base pulses to be totaled in counters one and three during the time the object breaks the upper and lower beams respectively. Taking the average of the times on counter one and three multiplied by the speed calculated using counter two yields an approximation of the width of the object passed through the beams.

\section{Laboratory Utilization}

This exercise provides several utilitarian options for instructor use in the lab. The first application of the exercise is to use it early in the introductory circuits class to introduce the students to the laboratory equipment, data taking and unit conversion techniques. In this 
application the circuit is constructed by the instructor or technician prior to the laboratory. Students then take data as instructed and calculate speed, and width as described in the work sheets shown in Figure 3.

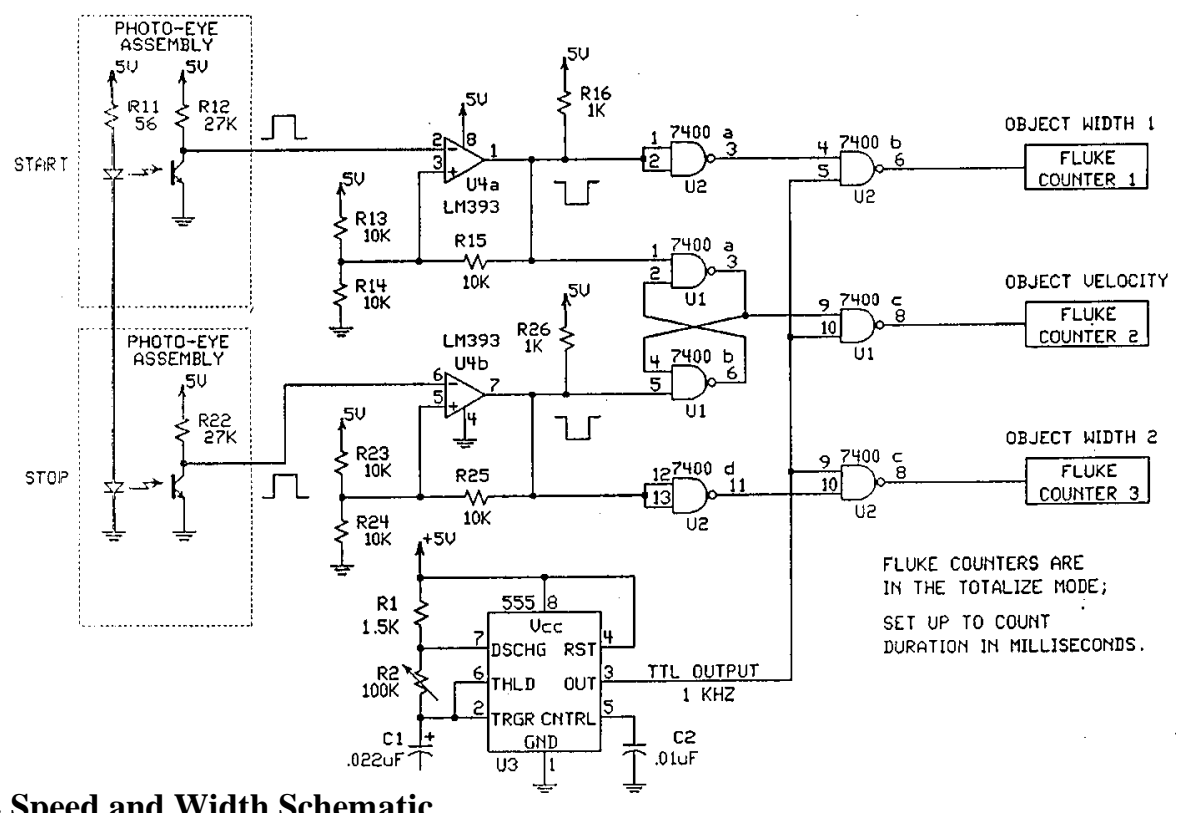

Figure 2 Speed and Width Schematic

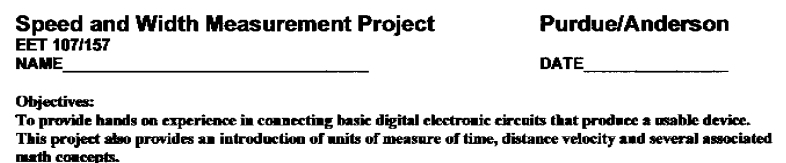

This project atso provides an iniroduction of mnits of measure of time, distance velocity and senthe device.

Measuring Speed and with of a moving Object

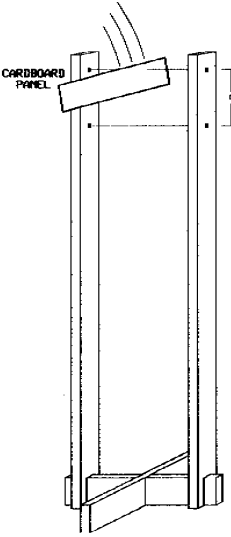

Figure 1

Figure 1 shows the apparatus for measuring speed and width of a moving object. A complete schematic of this circuit is attached. The switches that start and stop the timer in this application are irfrared infored devices emitter on one side, and delector on the chiner. The while at the same time reduce their sensitivity to the visible ambient light. Notice that the "switches" are separated by a measured fistance of 6 inches. This becomes the knounn distance in our speed calculations.

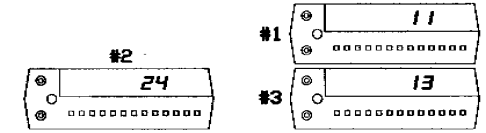$$
\text { Figure } 2
$$

Speed

We will use three counters for our experiments. Counter *2, shown in Figure 2 , measures the time in milliseconds it takes an object's bottom beam. We can calculate the speed at which an object passes through the bearns from the following formula:

Speed $=$ (distance) $\div$ (time)

Since the time is in ms which is $10^{3}$ (or 0.001 ) sec, and $d=6^{\prime \prime} / 12^{\prime \prime}$ )

$=0.5 \mathrm{ft}$

Speed $=\left[(0.5) \div\left(\right.\right.$ measured time in $\left.\left.m s \times 10^{-3}\right)\right]$ ftrsec

Sample Speed calculation: From the reading shown on counter $* 2, t=24 \mathrm{~ms}$

Speed $=\left[0.5 /\left(24 \times 10^{-3}\right)\right]=0.5 \mathrm{ft} / 0.024 \mathrm{sec}=20.83 \mathrm{ft} / \mathrm{sec}$

Width 1 in Figure 2 starts counting when an object breaks the first (upper) infrared bear and stops cous bingcting the firjt

Figure 3 Sample Data Sheets

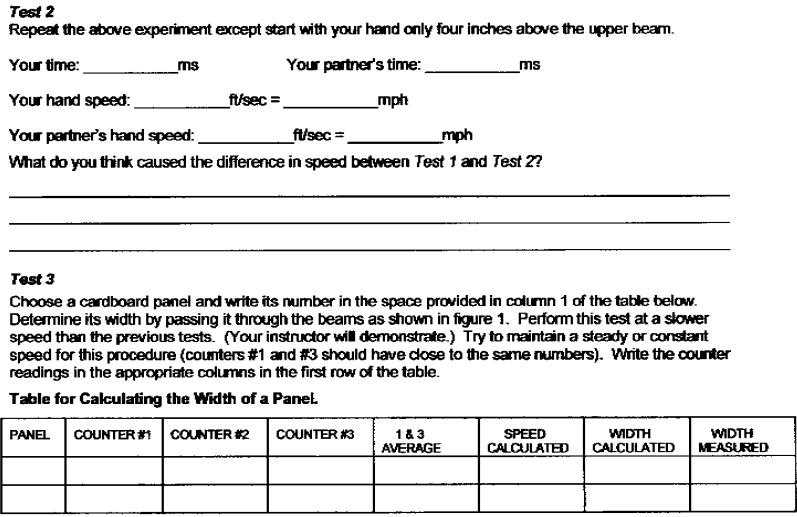

Repeat the test with another panel and write the data in the second row. Calculate the remaining data for Measure the witths of the cardboard panels with a ruler and insert the values in the final column of the

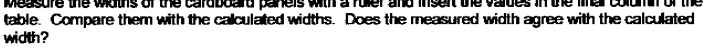

If not, what do you think might cause the error? 
This application of the exercise has also been successfully used several times in our Tech Prep program with a local high school. At the high school level it was determined that an extra prep period was required to prepare the students on making unit conversions and understanding the theories involved in the speed, time and distance relationships.

The second application of this exercise is in the first semester electronic circuits class (second semester in program). The instructor has the option of using it early in the class schedule as a packaged application illustrating digital and analog circuit applications. In this situation the students would be provided the circuit schematics and required to construct the circuit on proto boards, connect them to the test stands, take data and perform the calculations required to measure speed and width of various objects. A second option with this application is to delay the exercise until op-amps and comparators have been covered. In this scenario additional student tasks can be included such as calculating trip points, designing the time base and making numerous other calculations.

A third application currently under consideration for this exercise is to use it in the fourth semester electronic projects course as a start up project with planned guidance. In this case, block diagrams would be provided with suggested solutions from time to time to make sure that the project teams succeed in getting a working design in a one to three week period. This would provide early positive design experiences and considerable team building as they work towards the development of their semester project.

\section{Results}

The original test stand was built from wood with a base so that it rested on the floor. LED sensing beams were at a convenient height for the students to pass their hands and various objects through as they measured speed and width. Initial usage indicated that the exercise had a great deal of student appeal. Students initiated competitive efforts as to how fast objects could be passed through the uprights as well as the accuracy of their width measurements. Student interest and appreciation of the exercise was very good.

\section{Redevelopment}

Storage of the stands in our basement storage area over the summer pointed out a basic mechanical flaw in the design. The wood (soft pine) absorbed moisture and warped. This made alignment of the LED beams difficult. The wooden test stand is being redesigned to utilize PVC pipe for the uprights and have a new base structure to allow the stand to operate from the bench top rather than the floor. This design is intended to solve the warping experienced in the original and also make the exercise more portable for use as a demonstration activity.

A second area of concern also developed around the laboratory counters used in the original setup. The Fluke counters on our benches created problems for the students because of their high sensitivity. This created inconsistent test results in some cases and it was decided that a more reliable, less sensitive counter was needed. We are currently developing a counter display box to use with the circuit board and test stand that will utilize commercially available counters that do not require sensitivity adjustment by the students. A secondary goal of this development 
effort is to be able to use the new stand and associated equipment as a demonstration device for high school visitations.

Summary

This exercise achieves its goal of actively involving students in a technically challenging experience while providing a level of physical activity that creates interest and holds their attention. Laboratory projects such as the one described above enhance the learning process of EET students as well as providing the instructor with multiple options for laboratory use. To assure graduates are ready to solve real problems upon graduation, applications oriented laboratory exercises should be encouraged at all levels of engineering technology education

Bibliography

1)Malvino, Electronic Principles, Glencoe, 1995.

2)Pease, R.A. “Troubleshooting is More Effective With the Right Philosophy”, EDN, V34 January $5,1989$.

3)Matsuda, Electronic Troubleshooting, Prentice-Hall, 1992.

Russell A. Aubrey is an Associate Professor of EET at Purdue University, School of Technology at Anderson. His industrial background includes technical staff assignments with NASA-Langley Research Center, Texas

Instruments, Inc. and Seyberts/Anderson Electronics. Current teaching interests are analog and digital electronics. He is a member of ASEE and IEEE. 